\title{
A METHOD FOR FINDING A MINIMAL POINT OF THE LATTICE IN CUBIC NUMBER FIELDS (II)
}

\author{
By
}

Kan KaneKo

\begin{abstract}
We give a method for finding a minimal point adjacent to 1 of the reduced lattice in cubic number fields using an isotropic vector of the quadratic form and two-dimensional lattice.
\end{abstract}

\section{Introduction}

In the previous paper [3] with the same title, we proved six theorems which gave candidates of a minimal point adjacent to 1 in a reduced lattice $\mathscr{R}$.

In this paper we shall improve Theorem 6.1B, Theorem 6.2A and Theorem $6.3 \mathrm{~A}$ in [3]. We also give such an example that does not seem to occur very frequently in Theorem $6.3 \mathrm{~B}$ in [3]. We follow the notation and terminology used in the previous paper [3].

In the rest of this introduction, we shall show that $\phi_{10}$ need not be included in [3, Theorem $6.1 \mathrm{~B},(3)$,(ii-a)]. Also, we shall show that $\phi_{5}$ need not be included in $[3$, Theorem $6.2 \mathrm{~A},(2),(\mathrm{ii})]$.

THEOREM $6.1 \mathrm{~B}^{\prime} . \quad$ Let $\mathscr{R}=\langle 1, \lambda, \mu\rangle$ be a reduced lattice of $K$ such that $0<$ $\lambda<1,0<X_{\mu}<X_{\lambda}, 0<\omega_{1}(\lambda, \mu)<1, \omega_{2}(\lambda, \mu)>0, a>1,2|b|<1,0<\mu<1$, $\phi_{1}<1, F\left(\phi_{6}\right)<1$, where $a=F(\mu), b=Y_{\mu}$. Then

(1) If $F\left(\phi_{2}\right)<1$, then the minimal point adjacent to 1 is $\phi_{2}$.

(2) If $\phi_{2}>1, F\left(\phi_{2}\right)>1$, then the minimal point adjacent to 1 is $\phi_{6}$.

(3) If $\phi_{2}<1$ :

(i) if $b<0$, then the minimal point adjacent to 1 is $\phi_{6}$;

(ii) if $b>0$, then the minimal point adjacent to 1 is $\phi_{6}$ or $\phi_{9}$.

2010 Mathematics Subject Classification: 11R16, 11R27.

Key words and phrases: Cubic fields, Voronoi's algorithm, fundamental units.

Received April 28, 2014.

Revised August 25, 2014. 
Proof. (3) (ii) We assume that $b>0,2 \lambda+\mu<1$ and $\theta_{g}=\phi_{10}=3 \lambda+2 \mu$. Since $Y_{\lambda}<-1 / 2$ and $0<Y_{\mu}<1 / 2$, we have $Y_{3 \lambda+2 \mu}=3 Y_{\lambda}+2 Y_{\mu}<-3 / 2+1$ $=-1 / 2$. From this and $-1<Y_{3 \lambda+2 \mu}$, we have $0<Y_{1+3 \lambda+2 \mu}<1 / 2$. Hence, $F(1+3 \lambda+2 \mu)=Y_{1+3 \lambda+2 \mu}^{2}+Z_{1+3 \lambda+2 \mu}^{2}<Y_{3 \lambda+2 \mu}^{2}+Z_{3 \lambda+2 \mu}^{2}=F(3 \lambda+2 \mu)<1$. Since $F(1+3 \lambda+2 \mu)<1$ and $F\left(\phi_{6}\right)=F\left(-\phi_{6}\right)=F(-1-\lambda)<1$, by Remark 1.1 bellow, we have $F\left(\frac{1}{2}(-1-\lambda)+\frac{1}{2}(1+3 \lambda+2 \mu)\right)=F(\lambda+\mu)<1$. Therefore, since $0<$ $\lambda+\mu<1$ and $\mathscr{R}$ is a reduced lattice, the assumption such that $b>0,2 \lambda+\mu<1$ and $\theta_{g}=\phi_{10}$ leads to a contradiction. Hence, if $b>0,2 \lambda+\mu<1$, then $\theta_{g} \neq \phi_{10}$.

REMARK 1.1. If $F(\alpha)<1$ and $F(\beta)<1$, then $F(t \alpha+(1-t) \beta)<1$, where $\alpha, \beta \in K, 0 \leq t \leq 1(t \in \mathbf{Q})$.

THEOREM $6.2 \mathrm{~A}^{\prime}$. Let $\mathscr{R}=\langle 1, \lambda, \mu\rangle$ be a reduced lattice of $K$ such that $0<\lambda<1,0<X_{\mu}<X_{\lambda}, 0<\omega_{1}(\lambda, \mu)<1, \omega_{2}(\lambda, \mu)>0, a>1,2|b|<1, \mu>1$, $\phi_{1}>1$, where $a=F(\mu), b=Y_{\mu}$. Then

(1) If $F\left(\phi_{1}\right)<1$ :

(i) if $b<0$, then the minimal point adjacent to 1 is $\phi_{1}, \phi_{3}$ or $\phi_{4}$;

(ii) if $b>0$, then the minimal point adjacent to 1 is $\phi_{1}$ or $\phi_{7}$.

(2) If $F\left(\phi_{1}\right)>1, F\left(\phi_{6}\right)<1$, then the minimal point adjacent to 1 is $\phi_{6}$.

Proof. (2) From [3, Theorem 6.2A,(2),(ii)], suffice it to say that if $b>0$, then $\theta_{g} \neq \phi_{5}$. We assume that $F\left(\phi_{1}\right)>1, F\left(\phi_{6}\right)<1$. From $F\left(\phi_{1}\right)>1, F\left(\phi_{1}+1\right)$ $=F\left(\phi_{6}\right)<1$, by Lemma 2.1,(2) in Section 2, we have $Y_{\phi_{1}}<-1 / 2$. From this and $Y_{\mu}=b<1 / 2$, we have $Y_{\phi_{5}}=Y_{\phi_{1}+\mu-1}=Y_{\phi_{1}}+Y_{\mu}-1<-1 / 2+1 / 2-1=-1$. Hence, $F\left(\phi_{5}\right)>1$. Therefore, $\theta_{g} \neq \phi_{5}$.

\section{Preliminaries}

This section is a preparation for the next section.

Lemma 2.1. (1) $K \ni 1, \lambda, \mu$ are independent over $\mathbf{Q} \Rightarrow \omega_{2}(\lambda, \mu) \notin \mathbf{Q}$.

(2) Let $\alpha \in K \backslash \mathbf{Q}$. If $F(\alpha)>1, F(1+\alpha)<1$, then $Y_{\alpha}<-1 / 2$.

Proof. (1) Let $K=\mathbf{Q}(\theta), \theta^{3}+p \theta+q=0 \quad(p, q \in \mathbf{Q})$ and $\lambda=a_{1}+a_{2} \theta+$ $a_{3} \theta^{2}\left(a_{i} \in \mathbf{Q}\right), \mu=b_{1}+b_{2} \theta+b_{3} \theta^{2} \quad\left(b_{i} \in \mathbf{Q}\right)$. Then we have $Y_{\lambda}=\frac{1}{2}\left(2 a_{1}-2 p a_{3}-\right.$ 
$\left.a_{2} \theta-a_{3} \theta^{2}\right), Y_{\mu}=\frac{1}{2}\left(2 b_{1}-2 p b_{3}-b_{2} \theta-b_{3} \theta^{2}\right), \omega_{1}(\lambda, \mu)=-\frac{a_{2}-a_{3} \theta}{b_{2}-b_{3} \theta}$. From these and the definition of $\omega_{2}(\lambda, \mu)$, we obtain the following formula:

$$
\omega_{2}(\lambda, \mu)=\frac{1}{-b_{2}+b_{3} \theta}\left(\left|\begin{array}{ll}
a_{1} & a_{2} \\
b_{1} & b_{2}
\end{array}\right|+p\left|\begin{array}{ll}
a_{2} & a_{3} \\
b_{2} & b_{3}
\end{array}\right|+\left|\begin{array}{ll}
a_{3} & a_{1} \\
b_{3} & b_{1}
\end{array}\right| \theta+\left|\begin{array}{ll}
a_{2} & a_{3} \\
b_{2} & b_{3}
\end{array}\right| \theta^{2}\right) .
$$

Suppose that $\omega_{2}(\lambda, \mu) \in \mathbf{Q}$. Then from (2.1), we have

$$
-\omega_{2} b_{2}+\omega_{2} b_{3} \theta=\left|\begin{array}{ll}
a_{1} & a_{2} \\
b_{1} & b_{2}
\end{array}\right|+p\left|\begin{array}{ll}
a_{2} & a_{3} \\
b_{2} & b_{3}
\end{array}\right|+\left|\begin{array}{ll}
a_{3} & a_{1} \\
b_{3} & b_{1}
\end{array}\right| \theta+\left|\begin{array}{ll}
a_{2} & a_{3} \\
b_{2} & b_{3}
\end{array}\right| \theta^{2} .
$$

Since $1, \theta, \theta^{2}$ are independent over $\mathbf{Q}$, we have $\left|\begin{array}{ll}a_{2} & a_{3} \\ b_{2} & b_{3}\end{array}\right|=0$. From this and $\omega_{1}=-\frac{a_{2}-a_{3} \theta}{b_{2}-b_{3} \theta}$, we have $\omega_{1} \in \mathbf{Q}$. On the other hand, by [3, Proposition 2.2,(3)], $\omega_{1}(\lambda, \mu) \notin \mathbf{Q}$. Hence, we have reached a contradiction. Therefore, we have $\omega_{2}(\lambda, \mu) \notin \mathbf{Q}$.

(2) Since $F(1+\alpha)<1$, we have $-1<Y_{1+\alpha}<1$. Suppose that $Y_{\alpha}>-1 / 2$. Then $Y_{1+\alpha}=1+Y_{\alpha}>1 / 2$. From this, we have $1 / 4+Z_{1+\alpha}^{2}<Y_{1+\alpha}^{2}+Z_{1+\alpha}^{2}=$ $F(1+\alpha)<1$. Hence, $\left|Z_{1+\alpha}\right|<\sqrt{3} / 2$. Since $Y_{\alpha}>-1 / 2$ and $Y_{\alpha}<0$, we have $-1 / 2<Y_{\alpha}<0$. Hence, $F(\alpha)=Y_{\alpha}^{2}+Z_{\alpha}^{2}=Y_{\alpha}^{2}+Z_{1+\alpha}^{2}<1 / 4+3 / 4=1$. Since $F(\alpha)>1$, we have reached a contradiction. Therefore, we have $Y_{\alpha}<-1 / 2$.

Proposition 2.2. Let $\mathscr{R}=\langle 1, \lambda, \mu\rangle$ be a reduced lattice of $K$ such that $0<$ $\omega_{1}(\lambda, \mu)<1, \omega_{2}(\lambda, \mu)>0, a>1,2|b|<1,0<\mu<1, \phi_{1}>1$, where $a=F(\mu)$, $b=Y_{\mu}$. If $F\left(\phi_{2}\right)>1, F\left(\phi_{6}\right)<1$ :

(1) if $F\left(\phi_{1}\right)<1, b<0$, then the minimal point adjacent to 1 is $\phi_{1}$ or $\phi_{3}$;

(2) if $F\left(\phi_{1}\right)<1, b>0$, then the minimal point adjacent to 1 is $\phi_{1}$,

(3) if $F\left(\phi_{1}\right)>1$, then the minimal point adjacent to 1 is $\phi_{6}$.

Proof. We assume that $F\left(\phi_{2}\right)>1, F\left(\phi_{6}\right)<1$.

(a) By [3, Lemma 4.5,(1)], we have $\theta_{g} \in\left\{\psi_{i, y} ; y(\neq 0) \in \mathbf{Z}, 1 \leq i \leq 12\right\}$.

(b) We shall prove that $y \geq 1$. We note that $\left[y \omega_{i}\right] \leq y\left[\omega_{i}\right](y \leq-1)$ and that by $\left[3\right.$, Propositon 2.2,(3)] and Lemma 2.1,(1), $\left[-\omega_{i}\right]=-\left[\omega_{i}\right]-1$. We assume that $y \leq-1$. By [3, Remark 4.4,(1)], we have $\psi_{i, y} \leq \psi_{12, y}$.

The case $y \leq-2: \psi_{12, y}=\left[\omega_{2} y\right]+2+y \lambda+\left(\left[\omega_{1} y\right]+1\right) \mu \leq y\left[\omega_{2}\right]+2+y \lambda+$ $\left(y\left[\omega_{1}\right]+1\right) \mu=y\left(\left[\omega_{2}\right]+\lambda\right)+2+\mu \leq-2\left(\left[\omega_{2}\right]+\lambda\right)+2+\mu<\mu<1$. The case $y=$ $-1: \psi_{12,-1}=\left[-\omega_{2}\right]+2-\lambda+\left(\left[-\omega_{1}\right]+1\right) \mu=-\left[\omega_{2}\right]-1+2-\lambda=-\left[\omega_{2}\right]+1-\lambda=$ $-\left(\left[\omega_{2}\right]+\lambda\right)+1<0$. Therefore, if $y \leq-1$, then we have $\psi_{i, y} \neq \theta_{g}$. 
(c) We shall prove that $y=1$ or 2 . Since $\phi_{1}=\psi_{4,1}=\left[\omega_{2}\right]+\lambda>1$, for $y \geq 3$ we have $\psi_{i, y}=\left[\omega_{2} y\right]+j+y \lambda+\left(\left[\omega_{1} y\right]+k\right) \mu \geq y\left[\omega_{2}\right]+j+y \lambda+\left(y\left[\omega_{1}\right]+k\right) \mu \geq$ $2\left(\left[\omega_{2}\right]+\lambda+\left[\omega_{1}\right] \mu\right)+\left[\omega_{2}\right]+j+\lambda+\left(\left[\omega_{1}\right]+k\right) \mu=2\left(\left[\omega_{2}\right]+\lambda+\left[\omega_{1}\right] \mu\right)+\left[\omega_{2}\right]+1+$ $\lambda+\left[\omega_{1}\right] \mu+j-1+k \mu=2 \psi_{4,1}+\psi_{8,1}+j-1+k \mu>\psi_{8,1}$, where $-1 \leq j, k \leq 2$, $(j, k) \neq(2,-1),(2,2),(-1,-1),(-1,2)$. Therefore, if $y \geq 3$, then we have $\psi_{i, y} \neq \theta_{g} \quad(1 \leq i \leq 12)$.

(d) We shall prove that $y \neq 2$.

(i) The case $b<0$ : By [3, Lemma 4.5,(3),(i)], we have $\theta_{g} \in\left\{\psi_{1, y}, \psi_{3, y}, \psi_{4, y}\right.$, $\left.\psi_{5, y}, \psi_{8, y}, \psi_{9, y}, \psi_{10, y}, \psi_{12, y}\right\} . \quad \psi_{1,2}=\left[2 \omega_{2}\right]-1+2 \lambda+\left[2 \omega_{1}\right] \mu \geq 2\left[\omega_{2}\right]-1+2 \lambda=$ $\left(\left[\omega_{2}\right]+\lambda\right)-1+\left[\omega_{2}\right]+\lambda>\phi_{1}$. The case $F\left(\phi_{1}\right)<1$; By [3, Remark 4.4,(1)], we have $\psi_{i, 2}>\phi_{1}(i=1,3,4,5,8,9,10,12)$. Hence, $\psi_{i, 2} \neq \theta_{g}(i=1,3,4,5,8,9,10,12)$. The case $F\left(\phi_{1}\right)>1$; By $\left[3\right.$, Lemma 4.5,(10),(12)], we have $\psi_{1,2}=\left[2 \omega_{2}\right]-1+$ $2 \lambda+\left[2 \omega_{1}\right] \mu=2\left[\omega_{2}\right]+2 \lambda+\left[2 \omega_{1}\right] \mu=\left(\left[\omega_{2}\right]+\lambda\right)+\left[\omega_{2}\right]+\lambda+\left[2 \omega_{1}\right] \mu>\phi_{6}$. Hence, by [3, Remark 4.4,(1)], we have $\psi_{i, 2}>\phi_{6}(i=1,3,4,5,8,9,10,12)$. Therefore, $\psi_{i, 2} \neq \theta_{g}(i=1,3,4,5,8,9,10,12)$.

(ii) The case $b>0$ : By [3, Lemma 4.5,(3),(ii)], we have $\theta_{g} \in\left\{\psi_{2, y}, \psi_{4, y}, \psi_{5, y}\right.$, $\left.\psi_{6, y}, \psi_{7, y}, \psi_{8, y}, \psi_{9, y}, \psi_{11, y}\right\}$. We have $\psi_{4,2}=\left[2 \omega_{2}\right]+2 \lambda+\left[2 \omega_{1}\right] \mu \geq 2\left[\omega_{2}\right]+2 \lambda+$ $\left[2 \omega_{1}\right] \mu=\left[\omega_{2}\right]+\left(\left[\omega_{2}\right]+\lambda\right)+\lambda+\left[2 \omega_{1}\right] \mu>\psi_{8,1}=\phi_{6}$. From this and [3, Remark $4.4,(1)]$, we have $\psi_{i, 2}>\phi_{6}(i=4,5,6,7,8,9,11)$. By [3, Lemma 4.5,(12)], for $\psi_{2,2}=\left[2 \omega_{2}\right]-1+2 \lambda+\left(\left[2 \omega_{1}\right]+1\right) \mu$, there are four cases:

1) $\psi_{2,2}=2\left[\omega_{2}\right]+2 \lambda+2 \mu=\left[\omega_{2}\right]+\left(\left[\omega_{2}\right]+\lambda\right)+\lambda+2 \mu>\phi_{6}$.

2) $\psi_{2,2}=2\left[\omega_{2}\right]+2 \lambda+\mu=\left[\omega_{2}\right]+\left(\left[\omega_{2}\right]+\lambda\right)+\lambda+\mu>\phi_{6}$.

3) $\psi_{2,2}=2\left[\omega_{2}\right]-1+2 \lambda+2 \mu=\left(\left[\omega_{2}\right]+\lambda\right)-1+\left[\omega_{2}\right]+\lambda+2 \mu>\phi_{1}$.

4) $\psi_{2,2}=2\left[\omega_{2}\right]-1+2 \lambda+\mu=\left(\left[\omega_{2}\right]+\lambda\right)-1+\left[\omega_{2}\right]+\lambda+\mu>\phi_{1}$.

The case $F\left(\phi_{1}\right)<1$; we have $\psi_{2,2} \neq \theta_{g}$.

The case $F\left(\phi_{1}\right)>1$; Since $F\left(\left[\omega_{2}\right]+\lambda\right)>1, F\left(\left[\omega_{2}\right]+1+\lambda\right)<1$, by Lemma $2.1,(2)$, we have $Y_{\left[\omega_{2}\right]+\lambda}<-1 / 2$. From this we have $Y_{2\left[\omega_{2}\right]-1+2 \lambda+2 \mu}=2 Y_{\left[\omega_{2}\right]+\lambda}-$ $1+2 Y_{\mu}<-1-1+1=-1$. Hence, we have $F\left(2\left[\omega_{2}\right]-1+2 \lambda+2 \mu\right)>1$. Similarly, from $Y_{2\left[\omega_{2}\right]-1+2 \lambda+\mu}=2 Y_{\left[\omega_{2}\right]+\lambda}-1+Y_{\mu}<-1-1+1 / 2<-3 / 2$, we have $F\left(2\left[\omega_{2}\right]\right.$ $-1+2 \lambda+\mu)>1$. Hence, we have $\psi_{2,2} \neq \theta_{g}$. By (i), (ii), we conclude that $y \neq 2$.

(e) We shall prove (1), (2) and (3).

(i) The case $b<0$ : From (d), $\theta_{g} \in\left\{\psi_{1,1}, \psi_{3,1}, \psi_{4,1}, \psi_{5,1}, \psi_{8,1}, \psi_{9,1}, \psi_{10,1}, \psi_{12,1}\right\}$. By [3, Remark 4.4,(1)], $\phi_{6}=\psi_{8,1}<\psi_{9,1}<\psi_{10,1}<\psi_{12,1}$, so $\theta_{g} \in\left\{\psi_{1,1}, \psi_{3,1}, \psi_{4,1}\right.$, $\left.\psi_{5,1}, \psi_{8,1}\right\}$. From $F\left(\psi_{8,1}\right)<1$, we have $F\left(\psi_{1,1}\right)>1$. Therefore, we have $\theta_{g} \in$ $\left\{\psi_{3,1}, \psi_{4,1}, \psi_{8,1}\right\}$.

(1) If $F\left(\phi_{1}\right)<1$, then we have $\theta_{g}=\phi_{1}$ or $\phi_{3}$.

(3) We assume that $F\left(\phi_{1}\right)>1$. By [3, Lemma 4.5,(4)], $F\left(\psi_{3,1}\right)>F\left(\psi_{4,1}\right)>1$. Hence, we have $\theta_{g}=\phi_{6}$. 
(ii) The case $b>0$ : From (d), $\theta_{g} \in\left\{\psi_{2,1}, \psi_{4,1}, \psi_{5,1}, \psi_{6,1}, \psi_{7,1}, \psi_{8,1}, \psi_{9,1}, \psi_{11,1}\right\}$. By [3, Remark 4.4,(1)], $\phi_{6}=\psi_{8,1}<\psi_{9,1}<\psi_{11,1}$, so $\theta_{g} \in\left\{\psi_{2,1}, \psi_{4,1}, \psi_{5,1}, \psi_{6,1}\right.$, $\left.\psi_{7,1}, \psi_{8,1}\right\}$. By [3, Lemma 4.5,(9)], $F\left(\psi_{2,1}\right)>1$. Also, by [3, Lemma 4.5,(5)], $F\left(\psi_{7,1}\right)>1$. Therefore, we have $\theta_{g} \in\left\{\psi_{4,1}, \psi_{6,1}, \psi_{8,1}\right\}$. By [3, Lemma 4.2,(1)], we have $F\left(\psi_{6,1}\right)-F\left(\psi_{5,1}\right)=a\left(c_{1}+2\right)^{2}+2 b c_{2}\left(c_{1}+2\right)+c_{2}^{2}-a\left(c_{1}+1\right)^{2}-$ $2 b c_{2}\left(c_{1}+1\right)-c_{2}^{2}=2 c_{1} a+3 a+2 b c_{2}=2 a\left(c_{1}+1\right)+a\left(1+\frac{2 b}{a} c_{2}\right)>0$, where $c_{1}=$ $\left[\omega_{1}\right]-\omega_{1}, c_{2}=\left[\omega_{2}\right]-\omega_{2}$. Hence, $F\left(\psi_{6,1}\right)>F\left(\psi_{5,1}\right)$. From this and $F\left(\psi_{5,1}\right)>1$, we have $F\left(\psi_{6,1}\right)>1$. Therefore, $\theta_{g} \in\left\{\psi_{4,1}, \psi_{8,1}\right\}$. From this we have (2) $F\left(\phi_{1}\right)<$ $1 \Rightarrow \theta_{g}=\phi_{1}$ and (3) $F\left(\phi_{1}\right)>1 \Rightarrow \theta_{g}=\phi_{6}$.

COROLlaRy 2.3. Let $\mathscr{R}=\langle 1, \lambda, \mu\rangle$ be a reduced lattice of $K$ such that $0<$ $\omega_{1}(\lambda, \mu)<1, \omega_{2}(\lambda, \mu)>0, a>1,0<b<1 / 2,0<\mu<1, \phi_{1}>1$, where $a=F(\mu)$, $b=Y_{\mu}$. If $F\left(\phi_{2}\right)>1, F\left(\phi_{6}\right)<1$, then the minimal point adjacent to 1 is $\phi_{1}$ or $\phi_{6}$.

Remark 2.4. From the proof in [4, Theorem 2.1] and Proposition 2.2,(3), we can see that Theorem 6.1A in [3] does not require the assumption $0<X_{\mu}<X_{\lambda}, 0<\lambda<1$.

The following two lemmas are used to prove Lemma 3.1 in Section 3.

Lemma 2.5 ([5, Chapter 4, Section 2, p. 51]). Let $\mathscr{R}$ be a reduced lattice with the normalized basis $\{1, N, M\}$. If $\theta_{g}^{\tau}=(N+M)^{\tau}$, then $F\left(M_{(3)}\right)>1$.

Lemma 2.6 ([6, Lemma 4.3]). Let $\mathscr{R}$ be a reduced lattice. For $\alpha \in \mathscr{R}$ such that $F\left(\alpha_{(3)}\right)<1$, we define $\alpha_{*}:=\left\{\begin{array}{ll}\alpha_{(1)} & \text { if } F\left(\alpha_{(1)}\right)<1 \\ \alpha_{(2)} & \text { if } F\left(\alpha_{(1)}\right)>1 .\end{array}\right.$ Let $\alpha, \beta \in \mathscr{R}$ such that $X_{\alpha}>0,\left|Z_{\alpha}\right|<\sqrt{3} / 2, F(\beta)<1$. If $X_{\alpha}<X_{\beta}, Z_{\alpha} Z_{\beta}>0$, then $\alpha_{*}<\beta$.

\section{Improved form of the Theorem $6.3 \mathrm{~A}$ in [3]}

In this section we shall improve Theorem 6.3A,(1),(ii-a) and Theorem $6.3 \mathrm{~A},(2)$ in [3]. If we improve Theorem $6.3 \mathrm{~A},(2)$ in [3], we can further reduce the maximum number of candidates $\varphi \in \mathscr{R}$ such that we must check whether $F(\varphi)<1$ or not from at most four to at most three (see Remark 4.4).

To improve Theorem 6.3A,(1),(ii-a), we need the following lemma.

Lemma 3.1. Let $\mathscr{R}=\langle 1, \lambda, \mu\rangle$ be a reduced lattice of $K$ such that $0<\lambda<1$, $0<X_{\mu}<X_{\lambda}, 0<\omega_{1}(\lambda, \mu)<1, \omega_{2}(\lambda, \mu)>0, a>1,2|b|<1, \mu<0, \phi_{1}>1$, where 
$a=F(\mu), b=Y_{\mu}$. Then if $F\left(\phi_{1}\right)<1,\left[\omega_{2}\right]=1, \lambda+\mu<0$, then $\theta_{g} \neq 1+\phi_{9}=$ $1+2 \lambda+\mu$.

Proof. We assume that $F\left(\phi_{1}\right)<1,\left[\omega_{2}\right]=1, \lambda+\mu<0$ and $\theta_{g}=1+\phi_{9}$ $=1+2 \lambda+\mu$. We take a normalized basis $\{1, N, M\}$ of $\mathscr{R}$ and fix it. $1+$ $2 \lambda+\mu$ appears only in the following two cases of the proof of $[3$, Theorem 6.3A,(1)]:

1) $(1-2)$ in $\left[3\right.$, Table 1] i.e., $\psi_{1,2}\left(\omega_{1}>1 / 2\right)$,

2) (1-3) in [3, Table 1] i.e., $\psi_{1, d+1}(d=1)$.

We note that by [3, Theorem 3.6], $\lambda^{\tau}=N^{\tau},(N-M)^{\tau}$ or $M^{\tau}$. Moreover, by [3, Theorem 3.3], we see that $\lambda^{\tau}=(N-M)^{\tau} \Rightarrow \mu^{\tau}=-d N^{\tau}+(d+1) M^{\tau}$ and that $\lambda^{\tau}=M^{\tau} \Rightarrow \mu^{\tau}=N^{\tau}-d M^{\tau}$. In the case (1-2) in [3, Table 1], we have only one case that $\lambda^{\tau}=N^{\tau}, \mu^{\tau}=M^{\tau}$. In the case (1-3) in [3, Table 1], we have two cases, that is, $\lambda^{\tau}=(N-M)^{\tau}$ and $\lambda^{\tau}=M^{\tau}$. Hence, only the following three cases are possible:

(i) The case $\lambda^{\tau}=N^{\tau}, \theta_{g}^{\tau}=(2 N+M)^{\tau}, \omega_{1}(\lambda, \mu)>1 / 2$ which corresponds to (1-2) in [3, Table 1],

(ii) The case $\lambda^{\tau}=M^{\tau}, \theta_{g}^{\tau}=(N+M)^{\tau}, d(\lambda, \mu)=1$ which corresponds to (1-3) in [3, Table 1],

(iii) The case $\lambda^{\tau}=(N-M)^{\tau}, \theta_{g}^{\tau}=N^{\tau}, d(\lambda, \mu)=1$ which corresponds to (1-3) in [3, Table 1].

(i) The case $\lambda^{\tau}=N^{\tau}, \theta_{g}^{\tau}=(2 N+M)^{\tau}, \omega_{1}(\lambda, \mu)>1 / 2$ : From $\omega_{1}=\left|Z_{N}\right| /$ $\left|Z_{M}\right|>1 / 2$, we have $2\left|Z_{N}\right|>\left|Z_{M}\right|$. From this, we have $Z_{1+\lambda} Z_{1+2 \lambda+\mu}=Z_{N} Z_{2 N+M}$ $>0$. So, since $\left|Z_{1+\lambda}\right|=\left|Z_{N}\right|<\sqrt{3} / 2, F(1+2 \lambda+\mu)<1,0<X_{1+\lambda}<X_{1+2 \lambda+\mu}$ and $Z_{1+\lambda} Z_{1+2 \lambda+\mu}>0$, Lemma 2.6 leads to $(1+\lambda)_{*}<1+2 \lambda+\mu$. Since $F(\lambda)>1$, $F(1+\lambda)<1$, we see $1+\lambda=(1+\lambda)_{*}$. Hence, $1+\lambda<1+2 \lambda+\mu$. Therefore, this case is impossible.

(ii) The case $\lambda^{\tau}=M^{\tau}, \theta_{g}^{\tau}=(N+M)^{\tau}, d(\lambda, \mu)=1$ : By Lemma 2.5, this case is impossible.

(iii) The case $\lambda^{\tau}=(N-M)^{\tau}, \theta_{g}^{\tau}=N^{\tau}, d(\lambda, \mu)=1$ :

(a) The case $\left|Z_{\lambda}\right|<\sqrt{3} / 2$; Since $0<X_{1+\lambda}<X_{1+2 \lambda+\mu}, \quad Z_{1+\lambda} Z_{1+2 \lambda+\mu}=$ $Z_{N-M} Z_{N}>0, \quad\left|Z_{1+\lambda}\right|=\left|Z_{\lambda}\right|<\sqrt{3} / 2$, Lemma 2.6 leads to $1+\lambda=(1+\lambda)_{*}<$ $1+2 \lambda+\mu$. Therefore, $\theta_{g} \neq 1+2 \lambda+\mu$.

(b) The case $\left|Z_{\lambda}\right|>\sqrt{3} / 2$; Since $\left|Z_{1+\lambda}\right|=\left|Z_{\lambda}\right|>\sqrt{3} / 2, \quad F(1+\lambda)<1$, we have $\left|Y_{1+\lambda}\right|<1 / 2$. If $-1 / 2<Y_{1+\lambda}<0$, then $Y_{1+2 \lambda+\mu}<-1$, so $F(1+2 \lambda+\mu)>1$. Hence, we conclude that

$$
0<Y_{1+\lambda}<1 / 2
$$


Since $0<\lambda<1,-1 / 2<\mu<0$, we see $1 / 2<1+\lambda+\mu<1$. Hence, as $\mathscr{R}$ is a reduced lattice, we have

$$
F(1+\lambda+\mu)>1
$$

Since $1^{\tau}+\lambda^{\tau}=N^{\tau}-M^{\tau}, 1^{\tau}+2 \lambda^{\tau}+\mu^{\tau}=N^{\tau}$, we see

$$
M^{\tau}=\lambda^{\tau}+\mu^{\tau} \text {. }
$$

From (3.1), we have $-1<Y_{\lambda}<-1 / 2$ and $-1 / 2<Y_{1+\lambda+\mu}$. Hence, we see

$$
Y_{\lambda}<Y_{1+\lambda+\mu}
$$

Since $M^{\tau}$ is adjacent to $(N-M)^{\tau}$, we have

$$
\left|Z_{M}\right|<\left|Z_{N-M}\right| \text {. }
$$

If $\left|Y_{1+\lambda+\mu}\right|<\left|Y_{1+\lambda}\right|$, then by $\left|Z_{1+\lambda+\mu}\right|=\left|Z_{M}\right|<\left|Z_{N-M}\right|=\left|Z_{1+\lambda}\right|$, we obtain $F(1+\lambda+\mu)=Z_{1+\lambda+\mu}^{2}+Y_{1+\lambda+\mu}^{2}<Z_{1+\lambda}^{2}+Y_{1+\lambda}^{2}=F(1+\lambda)<1$. From this, by (3.2), we conclude that

$$
\left|Y_{1+\lambda+\mu}\right|>\left|Y_{1+\lambda}\right|
$$

If $Y_{1+\lambda+\mu}>0$, then we have $\left|Y_{1+\lambda+\mu}\right|<\left|Y_{1+\lambda}\right|$. From this, by (3.6), we conclude that

$$
Y_{1+\lambda+\mu}<0
$$

By (3.6), (3.7) and (3.1), we see $-Y_{1+\lambda+\mu}>1+Y_{\lambda}$, so $Y_{1+2 \lambda+\mu}<-1$. From this, $F(1+2 \lambda+\mu)>1$. Hence, $\theta_{g} \neq 1+2 \lambda+\mu$.

By (a), (b), this case is impossible. Therefore, by (i), (ii), (iii), the assumption leads to a contradiction.

THEOREM $6.3 \mathrm{~A}^{\prime}$. Let $\mathscr{R}=\langle 1, \lambda, \mu\rangle$ be a reduced lattice of $K$ such that $0<$ $\lambda<1,0<X_{\mu}<X_{\lambda}, 0<\omega_{1}(\lambda, \mu)<1, \omega_{2}(\lambda, \mu)>0, a>1,2|b|<1, \mu<0, \phi_{1}>1$, where $a=F(\mu), b=Y_{\mu}$. Then

(1) If $F\left(\phi_{1}\right)<1$, then the minimal point adjacent to 1 is $\phi_{1}$, $\phi_{2}$ or $\phi_{4}$.

(2) If $\phi_{2}>1$ :

(i) if $F\left(\phi_{1}\right)>1, F\left(\phi_{8}\right)<1$, then the minimal point adjacent to 1 is $\phi_{2}$ or $\phi_{8}$;

(ii) if $F\left(\phi_{1}\right)>1, F\left(\phi_{8}\right)>1, F\left(\phi_{6}\right)<1$, then the minimal point adjacent to 1 is $\phi_{6}$.

(3) If $\phi_{2}<1, F\left(\phi_{1}\right)>1, F\left(\phi_{6}\right)<1$, then the minimal point adjacent to 1 is $\phi_{6}$ or $\phi_{8}$. 
Proof. (1) is followed by [3, Theorem 6.3A,(1)] and Lemma 3.1.

(2) We assume that $\phi_{2}(\lambda, \mu)=\left[\omega_{2}(\lambda, \mu)\right]+\lambda+\mu>1$.

(i) We assume that $F\left(\phi_{1}\right)>1, F\left(\phi_{8}\right)<1$. We put $\lambda^{+}:=\lambda+\mu, \mu^{-}:=-\mu$.

(a) Since $\omega_{1}\left(\lambda^{+}, \mu^{-}\right)=-Z_{\lambda+\mu} / Z_{-\mu}=-\left(Z_{\lambda}+Z_{\mu}\right) /\left(-Z_{\mu}\right)=1-\omega_{1}(\lambda, \mu)$, we have $0<\omega_{1}\left(\lambda^{+}, \mu^{-}\right)=1-\omega_{1}(\lambda, \mu)<1$.

(b) Since $\omega_{2}\left(\lambda^{+}, \mu^{-}\right)=-Y_{\lambda^{+}}-\omega_{1}\left(\lambda^{+}, \mu^{-}\right) Y_{\mu^{-}}=-Y_{\lambda}-Y_{\mu}+\omega_{1}\left(\lambda^{+}, \mu^{-}\right) Y_{\mu}=$ $-Y_{\lambda}-Y_{\mu}+\left(1-\omega_{1}(\lambda, \mu)\right) Y_{\mu}=-Y_{\lambda}-\omega_{1}(\lambda, \mu) Y_{\mu}=\omega_{2}(\lambda, \mu)$, we have $\omega_{2}\left(\lambda^{+}, \mu^{-}\right)$ $=\omega_{2}(\lambda, \mu)>0$.

(c) $a\left(\mu^{-}\right)=F\left(\mu^{-}\right)=F(-\mu)=F(\mu)=a(\mu)>1$.

(d) $b\left(\mu^{-}\right)=Y_{\mu^{-}}=-Y_{\mu}=-b(\mu)$. From this and $-1 / 2<b(\mu)<0$, we have $0<b\left(\mu^{-}\right)<1 / 2$. Also from $-1 / 2<\mu<0$, we have $0<\mu^{-}<1 / 2<1$.

(e) Since $\phi_{2}\left(\lambda^{+}, \mu^{-}\right)=\left[\omega_{2}\left(\lambda^{+}, \mu^{-}\right)\right]+\lambda^{+}+\mu^{-}=\left[\omega_{2}(\lambda, \mu)\right]+\lambda=\phi_{1}(\lambda, \mu)$, we have $F\left(\phi_{2}\left(\lambda^{+}, \mu^{-}\right)\right)=F\left(\phi_{1}(\lambda, \mu)\right)>1$. Also, we have $\phi_{1}\left(\lambda^{+}, \mu^{-}\right)=\left[\omega_{2}\left(\lambda^{+}, \mu^{-}\right)\right]+$ $\lambda^{+}=\left[\omega_{2}(\lambda, \mu)\right]+\lambda+\mu=\phi_{2}(\lambda, \mu)$.

(f) Since $\quad \phi_{6}\left(\lambda^{+}, \mu^{-}\right)=\left[\omega_{2}\left(\lambda^{+}, \mu^{-}\right)\right]+1+\lambda^{+}=\left[\omega_{2}(\lambda, \mu)\right]+1+\lambda+\mu=$ $\phi_{8}(\lambda, \mu)$, we have $F\left(\phi_{6}\left(\lambda^{+}, \mu^{-}\right)\right)=F\left(\phi_{8}(\lambda, \mu)\right)<1$. With (a) to (f), Corollary 2.3 for $\mathscr{R}=\langle 1, \lambda, \mu\rangle=\left\langle 1, \lambda^{+}, \mu^{-}\right\rangle$leads to $\theta_{g}=\phi_{1}\left(\lambda^{+}, \mu^{-}\right)$or $\phi_{6}\left(\lambda^{+}, \mu^{-}\right)$. Hence, we have $\theta_{g}=\phi_{2}(\lambda, \mu)$ or $\phi_{8}(\lambda, \mu)$.

(ii) We assume that $F\left(\phi_{1}\right)>1, F\left(\phi_{8}\right)>1, F\left(\phi_{6}\right)<1$. By [3, Lemma 4.2,(1)], we have $F\left(\phi_{8}\right)-F\left(\phi_{2}\right)=F\left(\psi_{9,1}\right)-F\left(\psi_{5,1}\right)=a\left(c_{1}+1\right)^{2}+2 b\left(c_{1}+1\right)\left(c_{2}+1\right)+$ $\left(c_{2}+1\right)^{2}-a\left(c_{1}+1\right)^{2}-2 b\left(c_{1}+1\right) c_{2}-c_{2}^{2}=2 b\left(c_{1}+1\right)+2 c_{2}+1$, where $c_{1}=$ $\left[\omega_{1}\right]-\omega_{1}, c_{2}=\left[\omega_{2}\right]-\omega_{2}$. By [3, Lemma 4.5,(10)], we have $c_{2}<-1 / 2$. From this and $b<0$, we have $F\left(\phi_{8}\right)-F\left(\phi_{2}\right)=2 b\left(c_{1}+1\right)+2 c_{2}+1<0$. Therefore, we have $F\left(\phi_{2}\right)>F\left(\phi_{8}\right)$. From this and $F\left(\phi_{8}\right)>1$, we have $\theta_{g} \neq \phi_{8}, \phi_{2}$. Therefore, by $[3$, Theorem $6.3 \mathrm{~A},(2)]$, we have $\theta_{g}=\phi_{6}$.

(3) We assume that $\phi_{2}<1, F\left(\phi_{1}\right)>1, F\left(\phi_{6}\right)<1$. By [3, Theorem 6.3A,(2)], we have $\theta_{g}=\phi_{6}$ or $\phi_{8}$.

\section{Revised Main Theorems}

In this section, we shall summarize main theorems in [3, Section 6]. We also give an example such that $\theta_{g}=\phi_{6}+\phi_{9}=1+3 \lambda+\mu$.

For the simplicity, we denote the following conditions by $(\#)$ :

(\#) $0<\lambda<1,0<X_{\mu}<X_{\lambda}, 0<\omega_{1}(\lambda, \mu)<1, \omega_{2}(\lambda, \mu)>0, a>1,2|b|<1$, where $a=F(\mu), b=Y_{\mu}$.

THEOREM 4.1A. Let $\mathscr{R}=\langle 1, \lambda, \mu\rangle$ be a reduced lattice of $K$ such that (\#), $0<\mu<1, \phi_{1}>1$. Then 
(1) If $F\left(\phi_{1}\right)<1$ :

(i) if $b<0$, then the minimal point adjacent to 1 is $\phi_{1}, \phi_{3}$ or $\phi_{4}$;

(ii) if $b>0$, then the minimal point adjacent to 1 is $\phi_{1}$ or $\phi_{5}$.

(2) If $F\left(\phi_{1}\right)>1, F\left(\phi_{2}\right)<1$ :

(i) if $b<0$, then the minimal point adjacent to 1 is $\phi_{2}$;

(ii) if $b>0$, then the minimal point adjacent to 1 is $\phi_{2}$ or $\phi_{5}$.

(3) If $F\left(\phi_{1}\right)>1, F\left(\phi_{2}\right)>1, F\left(\phi_{6}\right)<1$, then the minimal point adjacent to 1 is $\phi_{6}$.

THeOREM 4.2A. Let $\mathscr{R}=\langle 1, \lambda, \mu\rangle$ be a reduced lattice of $K$ such that (\#), $\mu>1, \phi_{1}>1$. Then

(1) If $F\left(\phi_{1}\right)<1$ :

(i) if $b<0$, then the minimal point adjacent to 1 is $\phi_{1}, \phi_{3}$ or $\phi_{4}$;

(ii) if $b>0$, then the minimal point adjacent to 1 is $\phi_{1}$ or $\phi_{7}$.

(2) If $F\left(\phi_{1}\right)>1, F\left(\phi_{6}\right)<1$, then the minimal point adjacent to 1 is $\phi_{6}$.

THeOREM 4.3A. Let $\mathscr{R}=\langle 1, \lambda, \mu\rangle$ be a reduced lattice of $K$ such that (\#), $\mu<0, \phi_{1}>1$. Then

(1) If $F\left(\phi_{1}\right)<1$, then the minimal point adjacent to 1 is $\phi_{1}$, $\phi_{2}$ or $\phi_{4}$.

(2) If $\phi_{2}>1$ :

(i) if $F\left(\phi_{1}\right)>1, F\left(\phi_{8}\right)<1$, then the minimal point adjacent to 1 is $\phi_{2}$ or $\phi_{8}$;

(ii) if $F\left(\phi_{1}\right)>1, F\left(\phi_{8}\right)>1, F\left(\phi_{6}\right)<1$, then the minimal point adjacent to 1 is $\phi_{6}$.

(3) If $\phi_{2}<1, F\left(\phi_{1}\right)>1, F\left(\phi_{6}\right)<1$, then the minimal point adjacent to 1 is $\phi_{6}$ or $\phi_{8}$.

THeOREM 4.1B. Let $\mathscr{R}=\langle 1, \lambda, \mu\rangle$ be a reduced lattice of $K$ such that (\#), $0<\mu<1, \phi_{1}<1, F\left(\phi_{6}\right)<1$. Then

(1) If $F\left(\phi_{2}\right)<1$, then the minimal point adjacent to 1 is $\phi_{2}$.

(2) If $\phi_{2}>1, F\left(\phi_{2}\right)>1$, then the minimal point adjacent to 1 is $\phi_{6}$.

(3) If $\phi_{2}<1$ :

(i) if $b<0$, then the minimal point adjacent to 1 is $\phi_{6}$;

(ii) if $b>0$, then the minimal point adjacent to 1 is $\phi_{6}$ or $\phi_{9}$.

THEOREM 4.2B. Let $\mathscr{R}=\langle 1, \lambda, \mu\rangle$ be a reduced lattice of $K$ such that (\#), $\mu>1, \phi_{1}<1, F\left(\phi_{6}\right)<1$. Then the minimal point adjacent to 1 is $\phi_{6}$. 
THEOREM 4.3B. Let $\mathscr{R}=\langle 1, \lambda, \mu\rangle$ be a reduced lattice of $K$ such that (\#), $\mu<0, \phi_{1}<1, F\left(\phi_{6}\right)<1$. Then

(1) If $F\left(\phi_{8}\right)<1$, then the minimal point adjacent to 1 is $\phi_{8}$.

(2) If $F\left(\phi_{8}\right)>1$ :

(i) if $\phi_{9}<0$, then the minimal point adjacent to 1 is $\phi_{6}$ or $\phi_{6}+\phi_{9}$;

(ii) if $\phi_{9}>0$, then the minimal point adjacent to 1 is $\phi_{6}$ or $1+\phi_{9}$.

REMARK 4.4. From these six theorems above, we see that

(i) $\theta_{g} \in S:=\left\{\phi_{1}, \phi_{2}, \phi_{3}, \phi_{4}, \phi_{5}, \phi_{6}, \phi_{7}, \phi_{8}, \phi_{9}, 1+\phi_{9}, \phi_{6}+\phi_{9}\right\}$,

(ii) in each case of the theorems, the maximum number of candidates $\varphi \in S$ such that we must check whether $F(\varphi)<1$ or not is at most three.

ReMARK 4.5. In practical computation, if we take a $F$-point as $\lambda$, then we can change (2) in Theorem 4.1A as follows:

$(2)^{\prime}$ If $F\left(\phi_{1}\right)>1, F\left(\phi_{2}\right)<1, F\left(\phi_{6}\right)<1$, then the minimal point adjacent to 1 is $\phi_{2}$.

Indeed, by the proof of Theorem $6.2 \mathrm{~A}^{\prime}, F\left(\phi_{1}\right)>1$ and $F\left(\phi_{6}\right)<1$ imply that $\theta_{g} \neq \phi_{5}$.

Example 4.6. Let $K=\mathbf{Q}(\theta)$ be a cubic number field defined by $\theta^{3}-51589=0 \quad(\theta=37.22651403)$. Then $\mathscr{R}_{988}=\left\langle 1,\left(-3553-76 \theta+5 \theta^{2}\right) / 9912\right.$, $\left.\left(-1352+415 \theta-11 \theta^{2}\right) / 9912\right\rangle=\langle 1, \lambda, \mu\rangle . \quad 0<\lambda<1, \quad \mu<0 . \quad 0<X_{\mu}<X_{\lambda}$. $\omega_{1}(\lambda, \mu)=\frac{76+5 \theta}{415+11 \theta} . Y_{\lambda}=\frac{1}{2 c}\left(-7106+76 \theta-5 \theta^{2}\right)(c=9912) . Y_{\mu}=\frac{1}{2 c}(-2704-$ $\left.415 \theta+11 \theta^{2}\right) . \omega_{1}=0.31793235 . \quad Y_{\lambda}=-0.56526693 . \quad Y_{\mu}=-0.14674417 . \omega_{2}=$ 0.61192165 . Hence $\left[\omega_{2}\right]=0, \phi_{1}=\left[\omega_{2}\right]+\lambda=\lambda<1$.

(1) $N_{K / \mathbf{Q}}\left(x+y \theta+z \theta^{2}\right)=x^{3}-3 \times 51589 x y z+51589 y^{3}+51589^{2} z^{3}$.

(a) By (1), $\quad F\left(\phi_{6}\right)=F\left(\left[\omega_{2}\right]+1+\lambda\right)=F(1+\lambda)=F\left(\frac{1}{c}\left(6359-76 \theta+5 \theta^{2}\right)\right)$ $=\frac{1}{c^{2}} F\left(6359-76 \theta+5 \theta^{2}\right)=\frac{1}{c^{2}} \frac{N_{K / \mathbf{Q}}\left(6359-76 \theta+5 \theta^{2}\right)}{6359-76 \theta+5 \theta^{2}}=\frac{1}{c^{2}} \frac{941151982680}{6359-76 \theta+5 \theta^{2}}=$ $0.91591078<1$.

(b) By (1), $F(1+3 \lambda+\mu)=F\left(\frac{-2099+187 \theta+4 \theta^{2}}{c}\right)=\frac{1}{c^{2}} F(-2099+187 \theta+$ $\left.4 \theta^{2}\right)=\frac{1}{c^{2}} \frac{N_{K / \mathbf{Q}}\left(-2099+187 \theta+4 \theta^{2}\right)}{-2099+187 \theta+4 \theta^{2}}=\frac{1}{c^{2}} \frac{741426600096}{-2099+187 \theta+4 \theta^{2}}=0.72523368<1$.

(c) Since $-8458+263 \theta-\theta^{2}<0, \quad 2 \lambda+\mu=\frac{-8458+263 \theta-\theta^{2}}{c}<0$. From this $\phi_{8}=1+\lambda+\mu<1$. So $F\left(\phi_{8}\right)>1$. 
(d) Since $2 \lambda+\mu<0$, we have $1+3 \lambda+\mu<1+\lambda$. Therefore, by Theorem 4.3B,(2),(i), we have $\theta_{g}=1+3 \lambda+\mu=\phi_{6}+\phi_{9}$.

\section{Acknowledgment}

I appreciate the anonymous referee's valuable comments to improve this paper.

\section{References}

[1] B. Adam, Voronoi-algorithm expansion of two families with period length going to infinity, Math. Comp. 64 (1995), 1687-1704.

[2] B. N. Delone and D. K. Faddeev, The theory of irrationalities of the third degree, Transl. Math. Monographs, Vol. 10, Amer. Math. Soc., 1964.

[3] K. Kaneko, A method for finding a minimal point of the lattice in cubic number fields, Tsukuba J. Math. 38 (2014), 85-121.

[4] O. Lahlou and A. Farhane, Sur les points extrémaux dans un ordre cubique, Bull. Belg. Math. Soc. 12 (2005), 449-459.

[ 5] H. Wada, Applications of Computer to Number Theory, Sophia Kokyuroku in Mathematics, No. 7, 1980.

[6] H. C. Williams, G. Cormack and E. Seah, Calculation of the regulator of a pure cubic field, Math. Comp. 34 (1980), 567-611.

Institute of Mathematics

University of Tsukuba

1-1-1 Tennohdai, Tsukuba

Ibaraki 305-8573, Japan 